\title{
2-S14-4
}

\section{Optical inactivation of synaptic molecules by chromophore-assisted light inactivation}

\section{Kiwamu Takemoto}

Dept. Biochem. Mie Univ.

It is suggested that spatiotemporal dynamics of molecular activities such as locality and timing are important as well as simple ON / OFF system in the living cells. On the other hand, the physiological significance of these activities and the extent of their contribution to the in vivo remain largely unknown. Recently, live imaging has been used as a method to approach these issues. Although imaging technique has revealed the correlation between molecular and cellular dynamics and biological phenomena, new methods are needed to elucidate the causal relationship between them. The CALI (chromophore-assisted light inactivation) is one of the optical technologies for molecular inactivation by using photosensitizers that release reactive oxygen species upon irradiation. Such locally and temporally inactivation of target molecules by light is effective in elucidating the spatiotemporal properties of molecules, which are difficult to approach by genome manipulation. Our new CALI method is attracting a lot of attention as a next-generation technology for neuroscience (Humeau Y et al. Nat. Neurosci.2019, Frank JA et al. Nat. Biotechnol. 2019, Paoletti P et al. Nat. Rev. Neurosci. 2019 etc.). In this presentation, we will first introduce the elemental technology of our highly efficient and versatile CALI methods. In addition, the application of the CALI method to novel molecules will be introduced in detail, including our recently reported work on elucidating the memory mechanism using the CALI method (Takemoto K et al. Nat. Biotechnol. 2017). 